\section{Is it always Wilms' tumor? Localized cystic disease of the kidney in an infant: An extremely rare case report and review of the literature}

\author{
Christos Kaselas, Charikleia Demiri, \\ Vasilios Mouravas, Eleni Koutra, \\ Kleanthis Anastasiadis, \\ Ioannis Spyridakis
}

$2^{\text {nd }}$ Department of Pediatric Surgery, Aristotle University School of Medicine, "Papageorgiou" General Hospital, Thessaloniki, Greece

\begin{abstract}
Localized Cystic Disease of the Kidney (LCDK) is an extremely rare benign disease in pediatric population. Although its management is conservative and generally requires no treatment, the unfamiliarity with the disease can expose such patients to misdiagnosis as renal malignancies or uncertainty for proper treatment. We report such a case in an infant and review the current literature.
\end{abstract}

\section{Introduction}

Cystic lesions of the kidney are challenging cases for all specialties involved in the management and treatment of pediatric patients. Differential diagnosis varies and includes syndromic, genetic and non-syndromic cysts that can be either benign or malignant. Among them, Localized Cystic Disease of the Kidney (LCDK) represents one of the most infrequent diagnosis, with few cases described in the adult literature and even fewer described in children. ${ }^{1}$ Thorough knowledge of this condition is mandatory in order to avoid mistreatment that many times leads to unnecessary surgery. We report such a case in a 6-month-old child and review the relevant literature.

\section{Case Report}

An otherwise healthy patient, 6 months and 20 days old, one of two male twins of a healthy mother with no past medical or family history and of unknown father (IVF pregnancy from sperm donor), with an incidental finding of left kidney tumor on abdominal ultrasound, was referred to our department for further management and treatment. The patient was initially followed elsewhere for a scalp haemangioma and was investigated with abdominal ultrasound for other haemangiomas of the abdominal organs.

On admission, clinical examination revealed a palpable, soft, non-tender mass of the left abdomen. Baseline laboratory exams were normal, including $\beta$-Human chorionic gonadotropin, alpha-fetoprotein, and urine vanillylmandelic acid without any indication of renal function deterioration. In order to characterize the renal mass, an abdominal Magnetic Resonance Imaging (MRI) examination was performed on 3-Tesla Ingenia scanner, that identified a $3.6 \times 3.0 \times 2.0$ $\mathrm{cm}$ centrally located, mixed lesion of the left kidney with both cystic and solid characteristics, setting a differential diagnosis of either mesoblastic nephroma or Wilms' tumor (Figure 1). Coronal and axial T2 weighted sequences, fat suppressed T2 weighted in axial plane and axial respiratory T1 weighted sequence were carried out before the contrast infusion. The postcontrast fat suppressed axial T1 sequence indicated enhancement only in the septa of the lesion and in the normal kidney parenchyma. The diffusion weighted sequence revealed non recognizable restricted diffusion.

In view of those findings, we consulted with our pediatric oncology colleagues who proposed that this patient should be treated as a possible malignant tumor case, as malignancy is the most possible diagnosis in this age group. Chest computed tomography (CT) was normal and dimercaptosuccinic acid (DMSA) scan of the kidneys found the relative renal function of the left kidney to be $47 \%$ compared to $53 \%$ of the right one. The patient received preoperative chemotherapy followed by left radical nephrectomy according to the SIOP protocol.

Histological report came back as a surprise. The lesion was described as multiple cystic formations, extending to the renal cortex without penetrating the renal capsule. It was not encapsulated and there were areas of normal renal parenchyma between the cysts. No mitotic activity nor necrosis were found and lymph nodes sampling was normal. All the above characteristics were consistent with the diagnosis of LCDK (Figure 2).

The patient had a normal and uneventful post-operative period and was discharged under the care of both the pediatric surgical and pediatric nephrology team. At the moment, almost 1.5 years after the operation, follow up is satisfactory and the child remains healthy and asymptomatic.
Correspondence: Christos Kaselas, 2nd Department of Pediatric Surgery, Aristotle University School of Medicine, "Papageorgiou" General Hospital, Ring Road Nea Efkarpia, 56403, Thessaloniki, Greece.

Tel: +302313323548 .

E-mail: xkaselas@otenet.gr

iKey words: Localized Cystic Disease of the Kidney; Wilms' Tumor; Renal Cyst; Mesoblastic Nephroma; Autosomal Polycystic Kidney Disease.

Contributions: The authors contributed equally

Conflict of interest: The authors declare no potential conflict of interest.

Acknowledgements: The authors wish to thank Dr Gourvas Victoras, $\mathrm{MD}, \mathrm{PhD}$, Istolab Laboratory, for providing the histopathological report and images.

Funding: None.

Availability of data and materials: All data generated or analyzed are included in the text.

Ethics approval and consent to participate: The study follows all the ethical standards.

Informed consent: Informed consent was obtained.

Received for publication: 3 February 2020.

Revision received: 22 February 2020.

Accepted for publication: 24 February 2020.

This work is licensed under a Creative Commons Attribution NonCommercial 4.0 License (CC BY-NC 4.0).

(C) Copyright: the Author(s), 2020

Licensee PAGEPress, Italy

Pediatric Reports 2020; 12:8483

doi:10.4081/pr.2020.8483

\section{Discussion}

Localized Cystic Disease of the Kidney is an extremely rare benign entity in the pediatric population. ${ }^{1}$ It is so rare that it was not included in the consensus statement of an international working group on imaging of kidney cysts and cystic kidney diseases in children. ${ }^{2}$ According to our knowledge, our patient is the $11^{\text {th }}$ pediatric case reported in the international literature and he is the second youngest of all (Table 1). 1,3-10

Due to its rarity, differential diagnosis is challenging. Proper diagnosis will ensue proper conservative management with regular sequential imaging follow up. ${ }^{1,11}$ Lack of awareness of the disease and its 
specific clinical and imaging characteristics among adult-treating and pediatric-treating physicians may lead to misdiagnosis and mistreatment including unnecessary nephrectomy, as in our case.

LCDK is a non-familial, non-hereditary, non-progressive benign disease. 1,3,4,12 It is of unknown pathogenesis but the diagnosis in newborns and in infancy, as in our case suggest that it could possibly be congenital 10. It has a male predominance in adults and apparently in children as well (Table 1). ${ }^{11} \mathrm{It}$ may be asymptomatic or can present clinical signs and symptoms that are similar both in adults and children. Those would include flank, intermittent or acute abdominal pain, palpable abdominal mass, hematuria or proteinuria and hypertension. 1,3-10,11,13 Importantly, it is not characterized by renal insufficiency. ${ }^{11,12}$

Abdominal CT or MRI are the most reliable imaging modalities to set the diagnosis as specific characteristics have been described to differentiate LCDK from other renal cystic lesions. Thus, LCDK has a non-encapsulated multi-cystic mass appearance involving usually part of the kidney with the cysts being separated by normal or atrophic renal parenchyma. ${ }^{11,13}$ Normal excretion of contrast medium from the involved kidney is always present while there is no association with renal calculi. $1,11,13$

Pathological findings include variable sized cysts originating from dilated ducts and tubules extending from the renal medulla to the renal cortex that are separated by normal or atrophic renal parenchyma. ${ }^{10,14}$

Differential diagnosis is fairly wide and includes both benign and malignant conditions. The spectrum of benign cystic kidney diseases includes simple cysts, multicystic dysplastic kidneys, autosomal recessive or dominant polycystic kidney disease, and renal cysts that are part of other genetic and systemic diseases and syndromes (tuberous sclerosis, von HippelLindau syndrome, nephronophthisis, etc).2,13 Most commonly, LCDK would be confused with a hereditary polycystic kidney disease or multicystic dysplastic kidney disease. Thorough clinical and family history, laterality, deterioration of renal function, presence of cysts in other organs and disease-specific imaging characteristics should be sufficient to differentiate LCDK from other benign cystic kidney diseases. 1,11-13

However, apart from benign conditions, the most important diagnostic consideration is to rule out cystic or partially cystic neoplasms including cystic nephroblastoma, cystic nephroma, cystic neuroblastoma, clear cell sarcoma and mesoblastic nephroma, which are the most possible diagnoses, especially in infants. ${ }^{15}$ In such cases, tumor encapsulation with or without calcifications and displacement of normal renal parenchyma are the most helpful features that assist to distinguish malignancies from LCDK. $1,11-13$

Our case follows the typical pattern of misdiagnosis and mistreatment in pediatric cases of LCDK when treating physicians are not aware of the disease. It was investigated as a possible malignancy since the presence of a renal mass overwhelmed the lack of encapsulation, diagnosed and managed as one with final diagnosis to be established only after histopathological evaluation of the nephrectomy specimen. However, according
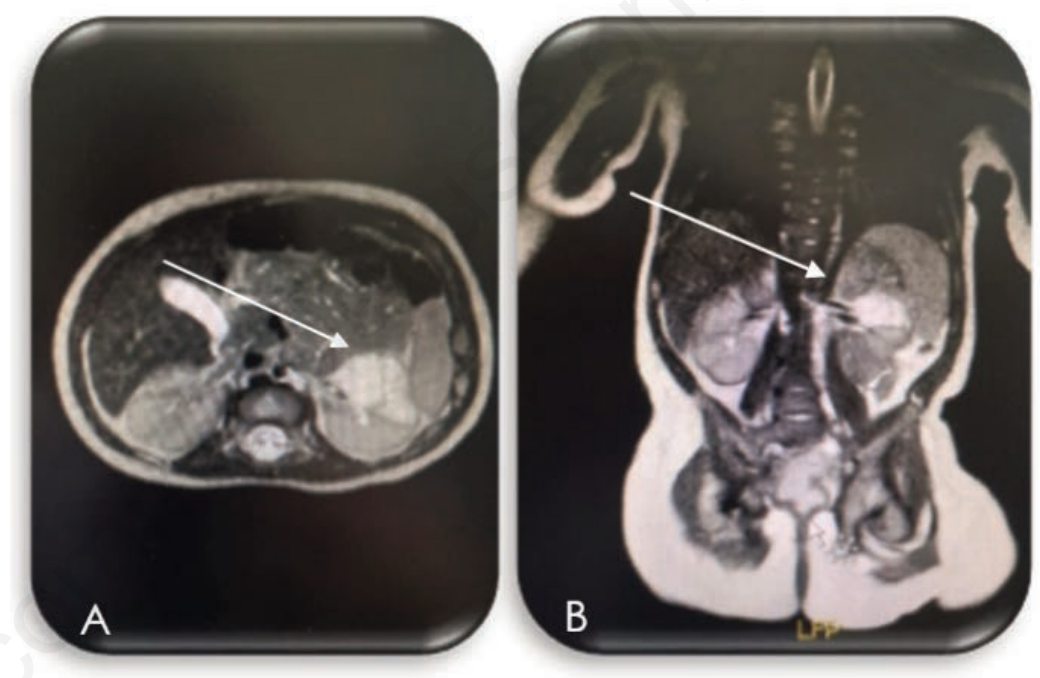

Figure 1. Axial (A) and Coronal (B) MRI image of the LCDK lesion of the left kidney (white arrows).
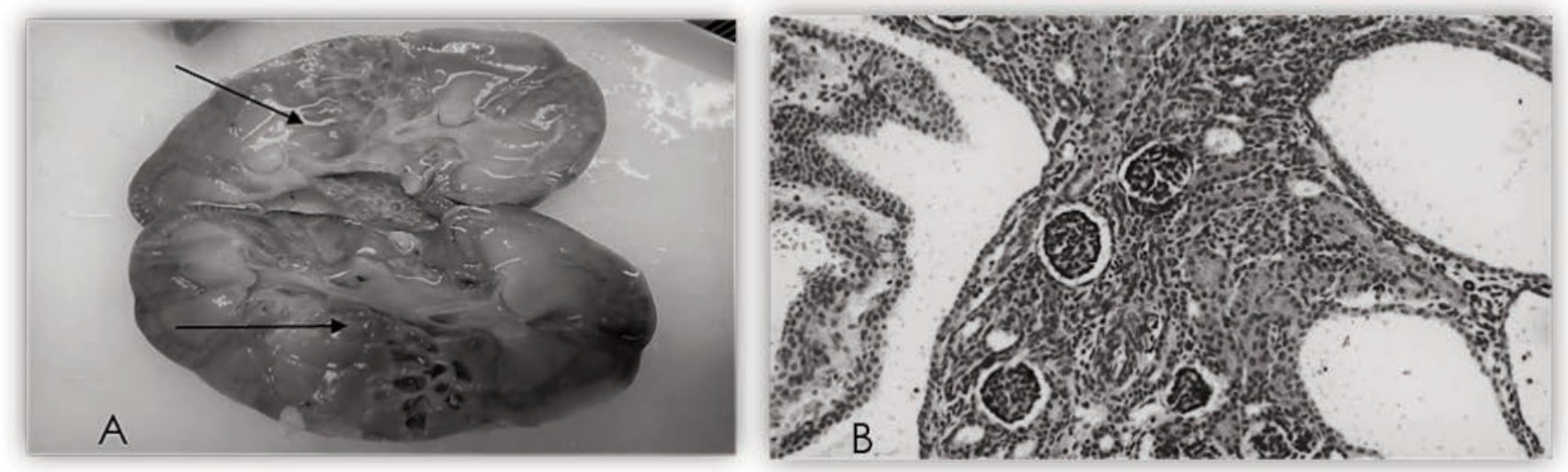

Figure 2. A. Gross histopathological specimen image demonstrating the cysts of the LCDK lesion (black arrows) B. Microscopic view after hematoxylin/eosin staining demonstrating normal renal parenchyma between the cysts. 
Table 1. Literature results of pediatric patients with localized cystic disease of the kidney.

\begin{tabular}{|c|c|c|c|c|c|}
\hline Publication & Age & Sex & Symptoms & Family History & Management \\
\hline Cho et al. 1979 & 3 years & Female & $\begin{array}{l}\text { Hematuria, Hypertension, } \\
\text { Abdominal mass }\end{array}$ & No & Surgical-Right Nephrectomy \\
\hline Levine et al. 1989 & 14 years & Not referred & Asymptomatic & No & Conservative \\
\hline Middlebrook et al. 1992 & 10 months & Not referred & Asymptomatic & No & Conservative \\
\hline Dugougeat et al. 2000 & 15 years & Male & $\begin{array}{l}\text { Flank pain, Borderline high blood pressure, } \\
\text { Orthostatic proteinuria }\end{array}$ & No & Conservative \\
\hline Dugougeat et al. 2000 & 10 years & Male & Right-sided abdominal pain & $\begin{array}{l}\text { Father suffered from } \\
\text { nephrolithiasis }\end{array}$ & Conservative \\
\hline Boybeyi et al. 2008 & 4.5 years & Female & $\begin{array}{l}\text { Urinary tract infection without } \\
\text { impairment of renal function }\end{array}$ & No & $\begin{array}{l}\text { Surgical } \\
\text { Total excision of the cyst }\end{array}$ \\
\hline Neyaz et al. 2012 & 16 years & Male & Acute abdominal pain in the periumbilical region & No & Conservative \\
\hline Solak et al. 2013 & 16 years & Male & Hypertension & No & $\begin{array}{l}\text { Surgical } \\
\text { Right hemi-nephrectomy }\end{array}$ \\
\hline Behr et al. 2016 & 6 years & Male & $\begin{array}{l}\text { One week of intermittent right-sided } \\
\text { abdominal pain }\end{array}$ & No & Surgical-Right nephrectomy \\
\hline Taskinen et al. 2009 & 1.5 months & Female & Neonatal Septicemia & Not mentioned & $\begin{array}{l}\text { Surgical } \\
\text { Left hemi-nephrectomy }\end{array}$ \\
\hline
\end{tabular}

to our literature review, this is the first case to receive pre-operative chemotherapy. This could be possibly attributed to the fact that other authors either did not worry of a malignancy or were not following SIOP protocol for pre-operative chemotherapy of renal tumors. 1,3,5,7,9,10 Nevertheless, despite the fact that it is reported that in patients receiving pre-operative chemotherapy for possible Wilms' tumor, 5.5\% will prove to have another malignancy and $1.6 \%$ a benign disease and although no chemotherapyrelated signs of toxicity have been reported in such cases, it is alarming for an infant to receive such unnecessary treatment. 16

\section{Conclusions}

In conclusion, it is apparent that due to its extreme rarity, the diagnosis of LCDK may be missed. As diagnosis of renal tumors is becoming more and more image-guided, first radiologists but also neonatologists, pediatricians and pediatric surgeons and urologists should become more aware of LCDK and its specific imaging characteristics and recognize it as a benign condition that will mostly need conservative management and regular follow up. This will help them reassure the parents but most importantly avoid unnecessary surgery.

\section{References}

1. Behr CA, Hesketh AJ, Williamson AK et al. Localized cystic disease of the kidney: A rare diagnosis of this uncommon condition in a child. J Pediatr Surg Case Reports 2016;12:41-3.

2. Gimpel C, Avni EF, Breysem L et al. Imaging of Kidney Cysts and Cystic Kidney Diseases in Children: An International Working Group Consensus Statement. Radiology 2019;290:769-82.

3. Cho KJ, Thornbury JR, Bernstein J et al. Localized cystic disease of the kidney: angiographic pathologic correlation. Am J Roentgenol 1979;132:891-5.

4. Levine E, Huntrakoon M. Unilateral renal cystic disease: CT findings. J Comput Assist Tomogr 1989;13:273-6.

5. Middlebrook PF, Nizalik E, Schillinger JF. Unilateral renal cystic disease: a case presentation. J Urol,1992;148:1221-3.

6. Dugougeat F, Navarro O, Soares Souza A et al. Multiple unilateral renal cysts in two children. Pediatr Radiol 2000;30: 346-8.

7. Boybeyi Ö, Karnak I, Orhan D et al. Cystic nephroma and localized renal cystic disease in children: diagnostic clues and management. J Pediatr Surg 2008;43:1985-9.

8. Neyaz Z, Kumar S, Lal $\mathrm{H}$ et al. Localized cystic disease of the kidney: A rare entity. J Radiol Case Rep 2012;6: 29-35.

9. Solak A, Gür M, Genç B et al. Localized cystic disease of the kidney: A rare cause of hypertension in a young adult. J Clin Imaging Sci 2013;3:33.

10. Taskinen S, Lohi J, Kivisaari R et al. Segmental cystic kidney tumours in children. Scand J
Urol Nephrol 2009;43: 476-81.

11. Slywotzky CM, Bosniak MA. Localized cystic disease of the kidney. Am J Roentgenol 2001;176:843-9.

12. Ding Y, Chen L, Deng FM et al. Localized cystic disease of the kidney: Distinction from cystic neoplasms and hereditary polycystic diseases. Am J Surg Pathol 2013;37:506-13.

13. Dowden EE, Osunkoya AO, Baumgarten DA. Localized Cystic Disease of the Kidney: An Unusual Entity That Can Mimic a Cystic Neoplasm. Am J Kidney Dis 2010;55:609-13.

14. Kim DJ, Kim MJ. Localized cystic disease of the kidney: CT findings. Abdom Imaging 2003;28:588-92.

15. van den Heuvel-Eibrink MM, Grundy P, Graf N, et al. Characteristics and survival of 750 children diagnosed with a renal tumor in the first seven months of life: A collaborative study by the SIOP/GPOH/SFOP, NWTSG, and UKCCSG Wilms tumor study groups. Pediatr Blood Cancer 2008;50:1130-4.

16. Tournade MF, Com-Nougue C, de Kraker $\mathrm{J}$ et al. Optimal duration of preoperative chemotherapy in unilateral non metasttic Wilms tumor in children older then six months. Results of the ninth International Society of Pediatric Oncology tumor trial. J Clin Oncol 2001;19:488-500.ù 